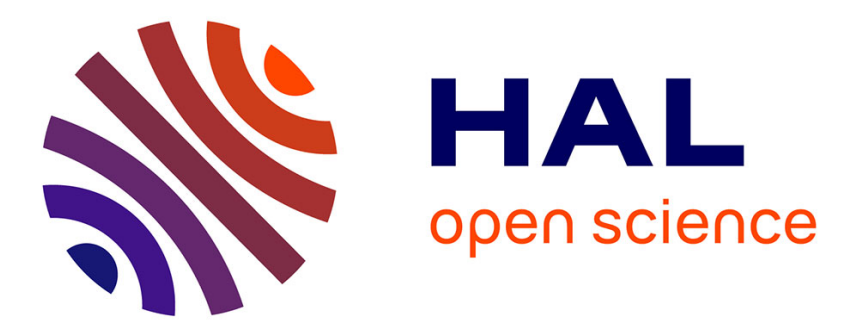

\title{
Optimum image processing for morphological study of granular films
}

\author{
A. Beghdadi, A. Constans, P. Gadenne, J. Lafait
}

\section{To cite this version:}

A. Beghdadi, A. Constans, P. Gadenne, J. Lafait. Optimum image processing for morphological study of granular films. Revue de Physique Appliquée, 1986, 21 (1), pp.73-80. 10.1051/rphysap:0198600210107300 . jpa-00245413

\section{HAL Id: jpa-00245413 https://hal.science/jpa-00245413}

Submitted on 1 Jan 1986

HAL is a multi-disciplinary open access archive for the deposit and dissemination of scientific research documents, whether they are published or not. The documents may come from teaching and research institutions in France or abroad, or from public or private research centers.
L'archive ouverte pluridisciplinaire HAL, est destinée au dépôt et à la diffusion de documents scientifiques de niveau recherche, publiés ou non, émanant des établissements d'enseignement et de recherche français ou étrangers, des laboratoires publics ou privés. 


\title{
Optimum image processing for morphological study of granular films
}

\author{
A. Beghdadi $\left({ }^{+\times}\right)$, A. Constans $\left({ }^{+}\right)$, P. Gadenne $\left({ }^{\times}\right)$and J. Lafait $\left({ }^{\times}\right)$ \\ $\left({ }^{+}\right)$Groupe d'analyse d'images biomédicales (CNAM, Paris 5), 3, Bd Pasteur, 75015 Paris, France \\ ( ${ }^{\times}$Laboratoire d'Optique des Solides, U.A. 040781 du C.N.R.S., Université Pierre et Marie Curie 4, place Jussieu, \\ 75252 Paris Cedex 05, France
}

(Reçu le 4 octobre 1985, accepté le 4 octobre 1985)

\begin{abstract}
Résumé. - La binarisation des clichés de microscopie électronique par transmission de couches minces granulaires est une étape indispensable pour l'étude statistique de la morphologie de la couche, dans le cadre des lois d'échelle et de la théorie fractale. Nous présentons un processus de traitement d'image conduisant à un seuil optimal de binarisation, sans perte d'information. Ce traitement, efficace même sur des films présentant un histogramme de niveaux de gris unimodal, est testé sur différentes couches minces granulaires de métal ou de composés métaldiélectrique.
\end{abstract}

\begin{abstract}
The binarization of transmission electron micrographs of thin granular films is an essential step for the statistical study of the film morphology in the framework of scaling laws and fractal theory. An image processing is developed, leading to an unambiguous optimum binarization threshold, without any loss of information. This treatment, efficient even on films with unimodal gray level histograms, is tested on different granular metallic or metal-dielectric films
\end{abstract}

\section{Introduction.}

The granular media attract presently a renewed interest in many fields : astronomy, meteorology, petrology, biology, chemistry, heliothermal conversion [1]... An exact knowledge of the morphology of these media, directly tractable on a computer, is essential for any further statistical treatment : for instance, in the determination of the critical exponents and fractal dimension [2] deduced from a statistical study of the cluster morphology at the percolation (perimeter, surface, distances...), or in the development of new optical theories of the dielectric function of these media, based on their real structure [3].

The source of information concerning the morphology is generally a Transmission Electron Micrograph (TEM) of a thin film (about a few $100 \AA$ thickness). This information can be stored on a magnetic support after digitization of the micrograph. The statistical study of the morphology can be achieved only on a binary image (with two gray levels), while the digitized micrograph usually covers an extended gray level range. The binarization process must, at least, preserve the information and, if possible, improve the micrograph quality. The main quality criterion which we use is the comparison between the metal volume fraction (in 2D) obtained by digital image processing and the one determined by using other methods of characterization (Rutherford Backscattering, microprobe analysis...) whenever they can be performed. A secondary criterion is the existence of an infinite cluster after image treatment in percolating systems.

Our aim in this paper is to point out the importance of the image treatment leading to the image binarization, which is essential to any statistical study of the image morphology. We present the different steps of the TEM micrograph treatment up to the binary image of the digitized film. We develop the process according to the following sequence :

$\S$-2 Digitization of the micrograph with a microdensitometer.

$\$$-3 Correction of non-homogeneous illumination effects and filtering (if necessary).

§-4 Selection of thresholding methods for binarization and (eventually) contrast enhancement. $\S-5$ Validity of the image treatment.

The different methods are tested out on real TEM micrographs of granular metallic or metal/dielectric 
thin films prepared by thermal evaporation $(\mathrm{Au})$, or sputtering $\left(\mathrm{Pt}-\mathrm{Al}_{2} \mathrm{O}_{3}\right)$. Other results obtained on these films and concerning percolation, fractal, theory and optical properties will be published elsewhere.

\section{Digitalization of the micrograph.}

A micrograph can be considered as a continuous distribution of gray levels. The transfer of this image information to the computer supposes that it has been digitized, i.e., that a matrix of discrete windows has been defined on the micrograph and that a gray level has been attributed to each window. For this purpose, we use the Optronics Photoscan P1000 scanner system which associates to each density point (window) an integer, creating thus a matrix of $512 \times 512$ points on the image. Each point is coded on 8 bits, allowing the density range to be divided into 256 gray levels. The aim of a good sampling is to represent a continuous micrograph by a finite array $(M \times N)$ of numbers (0 to 255), called samples, without any loss of information. This can be achieved by using the well-known Whittaker-Kotelnikov-Schanon theorem [4]. Let us define the digitization function as follows : to a picture element (pixel) $x_{k l} \in I_{(M \times N)}$ is associated the number $y_{j}=f\left(x_{k l}\right), I_{(M \times N)}$ being the image of size $M \times N$. If $\omega_{c}$ is the limit frequency (related to the smallest detail to be resolved) in the image, one can determine the optimal sampling period $T$ by satisfying the condition :

$$
T \leqslant \frac{1}{2 \omega_{\mathrm{c}}} \cdot\left(\omega_{\mathrm{c}}=1 / T_{\mathrm{c}}\right)
$$

In our case the thin film image is magnified by the electron microscope. If $G$ is the magnification and $r$ the resolution of the microscope, the limit frequency is then :

$$
\omega_{\mathrm{c}}=\frac{1}{r G}
$$

so we deduce the sampling condition :

$$
T \leqslant \frac{r G}{2} \text {. }
$$

In our images, $r$ is about $15 \AA$ and $G$ between 30000 and 100000 , therefore the good sampling period must satisfy :

$$
T \leqslant 25 \mu \mathrm{m} \quad(G=30000)
$$

or

$$
75 \mu \mathrm{m} \quad(G=100000)
$$

depending on the magnification. In each case we have selected the adequate raster size as a function of the upper condition.

\section{Correction of non homogeneous illumination effects and filtering.}

These effects can arise due to a non uniform illumination in the microscope. The principle of the correction is to assign the same mean gray level to adequate windows in the image. The digitized picture of size $M \times N$, where $M$ is the number of rows and $N$ the number of pixels in a row of scanning, is divided into elementary windows of size $m \times n$ (with $1<n<N$ and $1<m<M$ ). The dimension $m \times n$ must be large enough to avoid modifications of the cluster morphology and small enough to take into account the illumination inhomogeneities. In each window $W_{i}$, we compute the mean gray level $\mu_{i}(i$ satisfies $1 \leqslant i \leqslant L$ where $L$ is the number of windows) by analysing the gray level histogram $h_{i}$ of the $i$ th window.

Then :

$$
\mu_{i}=\left\{\sum_{x_{k l} \in W_{i}} y_{j} \cdot h_{i}\left(y_{j}\right)\right\} / \sum_{x_{k l} \in W_{i}} h\left(y_{j}\right)
$$

where $y_{j}=f\left(x_{k l}\right)$ is the gray level associated to the pixel $x_{k l}$ of the $k$ th row and $l$ th column.

We eventually consider all windows ( $i$ varying from 1 to $L$ ) making the entire picture, and transform each considered pixel of gray level $y_{j}$ into $y_{j}^{\prime}$ according to :

where

$$
y_{j}^{\prime}=\alpha_{i} y_{j}
$$

$$
\alpha_{i}=\frac{\mu_{t}}{\mu_{i}}
$$

$\mu_{t}$ being the mean gray level of the entire image, and $\mu_{i}$ the mean gray level of the window containing the considered pixel.

The effect of this treatment is illustrated in figures 1 (a, b, c, d). We applied this process to all our images before any other operation. It improves the pictures without modifying the contours. One can notice that the values of the $\alpha$ coefficient allow an evaluation of the micrograph quality.

Filtering is used in order to eliminate image impurities (dust or defects which may be introduced during the development of the film. The sampling period $(T=100 \mu \mathrm{m})$ being comparable to the period of this noise, we use a median filter $(3 \times 3$ or $5 \times 5)$. Contrarily to some other filters, which blur the object frontiers, this filter does not .modify the contours [4].

\section{Contrast enhancement and selection of a thresholding method for binarization.}

4.1 Gray LeVEL histogram STUDY. - This function describes the gray level distribution in the image. It is useful for the classification of objects in a picture [9-11]. In our case of granular films, the purpose is to separate the metal from the dielectric $[12,13]$. Several authors have developed a large variety of thresholding methods based on the histogram information [14-17]. In this work, we consider two types of histograms (Fig. 2), bimodal histogram (two peaks) and unimodal histogram (one peak), corresponding to our experimental cases.

4.2 Thresholding FUnCTION. - Let $x_{k l}$ be a pixel with an associated gray level $y_{j}=f\left(x_{k l}\right)$, where $f$ 


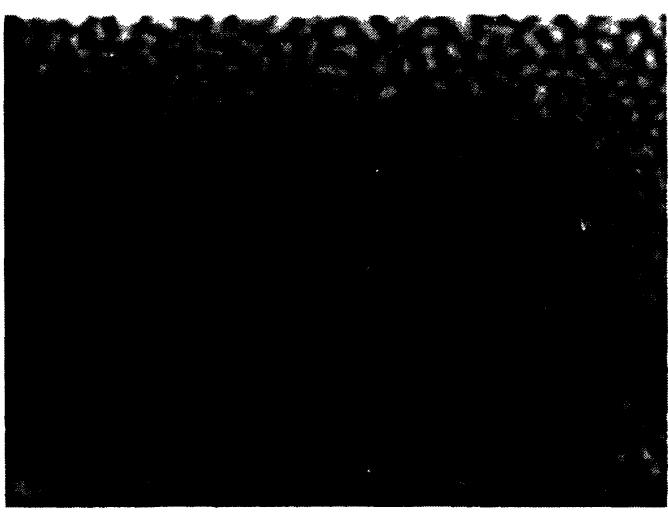

FIGURE 1a

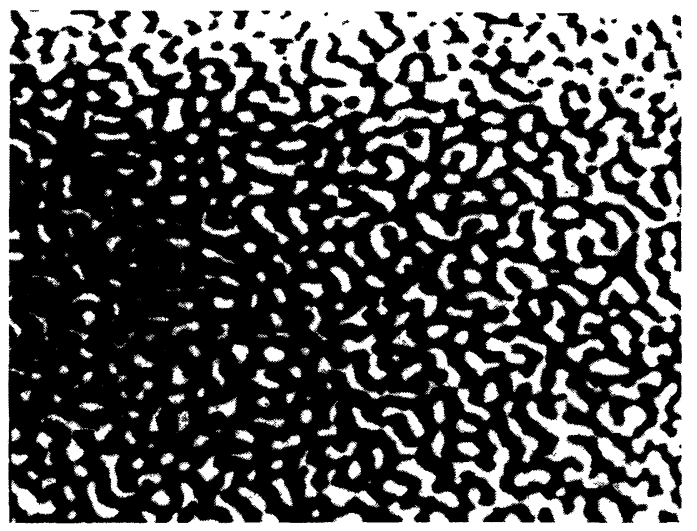

FIGURE 1c

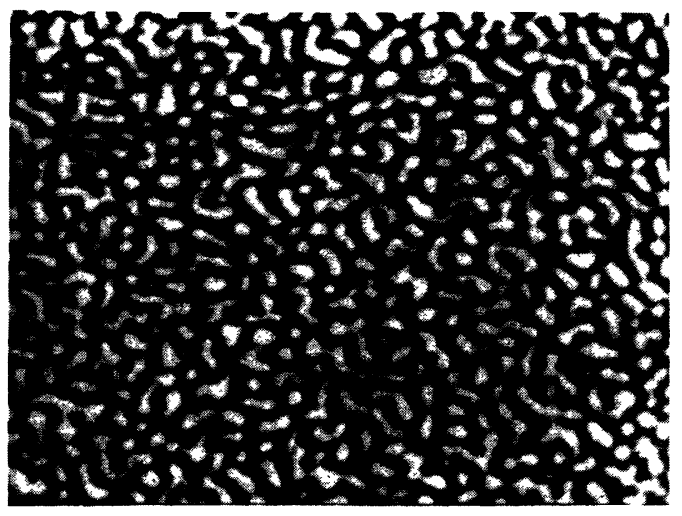

FIGURE 1b

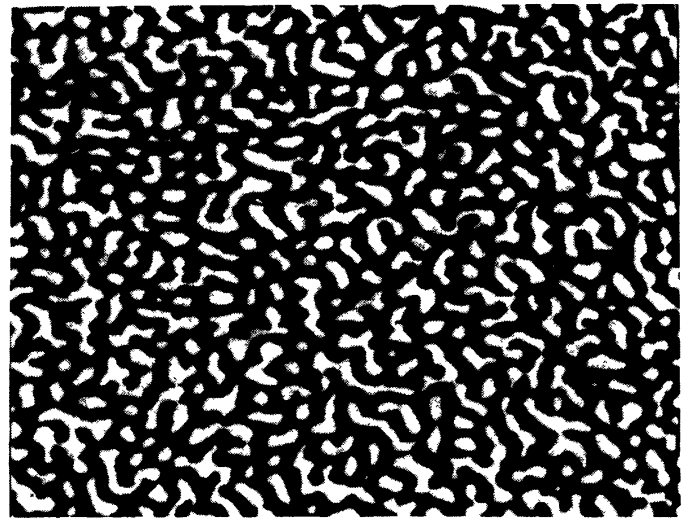

Figure 1d

Fig. 1. - Image of a thin granular film $\left(\mathrm{Pt}-\mathrm{Al}_{2} \mathrm{O}_{3}\right)$. a : original image (image $\left.\mathrm{a}\right) . \mathrm{b}:$ result of non homogeneous illumination effect correction (image b). $c$ : result of the thresholding of the image a. $d:$ result of the thresholding of the image $b$.

is the sampling function. $y_{j}$ lies on a certain gray level scale $\left(y_{\min }, y_{\max }\right)$. The thresholding function transforms the initial image $I_{(M \times N)}$ into the thresholded image $I_{(M \times N)}^{\prime}$ by assigning the same gray level (in practice 0 or 1) to a first set of pixels with gray levels less or equal to the gray level threshold $t$ and another value (in pratice 1 or 0 ) to the complementary set of pixels. This transformation is defined by :

$$
\begin{array}{lll}
T_{t}\left(y_{j}\right)=0 & \text { if } & y_{j} \leqslant t \\
T_{t}\left(y_{j}\right)=1 & \text { if } & y_{j}>t
\end{array}
$$

In other words, if $x_{k l} \in I_{(M \times N)}$ with gray level $y_{j}=f\left(x_{k l}\right)$, then $x_{k l}^{\prime} \in I_{(M \times N)}^{\prime}$ is defined by :

$$
x_{k l}^{\prime}=(T \circ f)\left(x_{k l}\right) .
$$

It is not obvious to select the gray level threshold $t$. Two cases can be distinguished by considering the gray level histogram :

4.2.1 Bimodal histogram. - The bimodality of a gray level histogram clearly points out that two classes are present in the image. In this case, it is not difficult to select the threshold if the valley is narrow enough [18]. When the profile of the histogram suggests more than one threshold candidate, one can approximate the histogram by two Gaussian distributions. It is then possible to express the misclassification error in terms of the Gaussian parameters [18]. By minimizing this error, one can find the optimal threshold. However it is not always obvious to fit the histogram by two Gaussians. For instance in Granlung's method [19], the inverse of a characteristic matrix is not always defined, whereas in Polak-Ribierre method [20], a null gradient cannot always be reached.

4.2.2 Unimodal histogram. - This behaviour is often met in our images. It was also observed by Rosenfeld et al. [10] in the flir images of tank. Unimodality suggests the presence of a single class. But the visual perception distinguishes two different structures.

Contrast enhancement. - The problem is to separate these two structures. The conventional contrast enhancement methods $[6,24,25]$ do not allow the distinction between two classes in an unimodal histogram. Therefore we developed a contrast enhancement technique based on a local analysis of gray levels. In the meantime, Gordon et al. [5] have proposed a similar method, which we ultimately prefer to use with some modifications. We recall this method and we explain in 


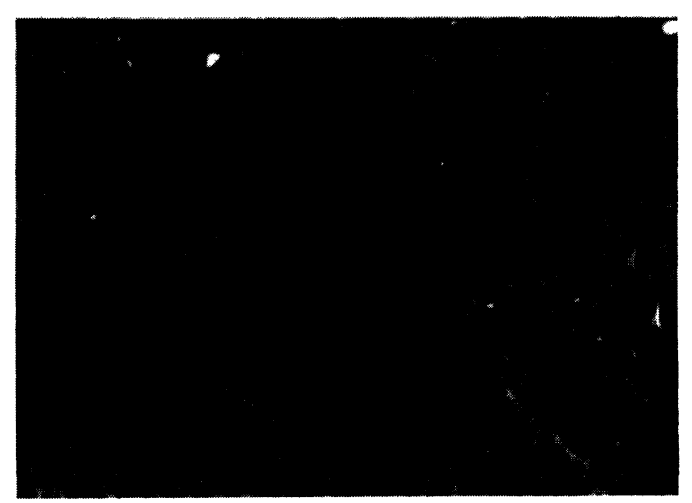

FIGURE 2a

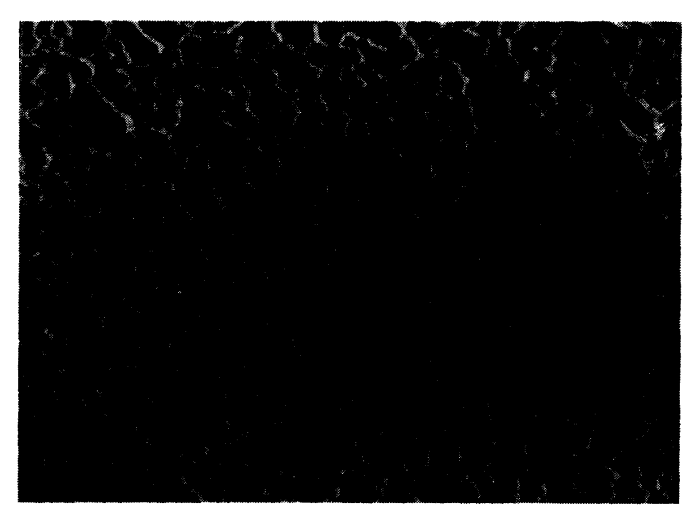

Figure 2b

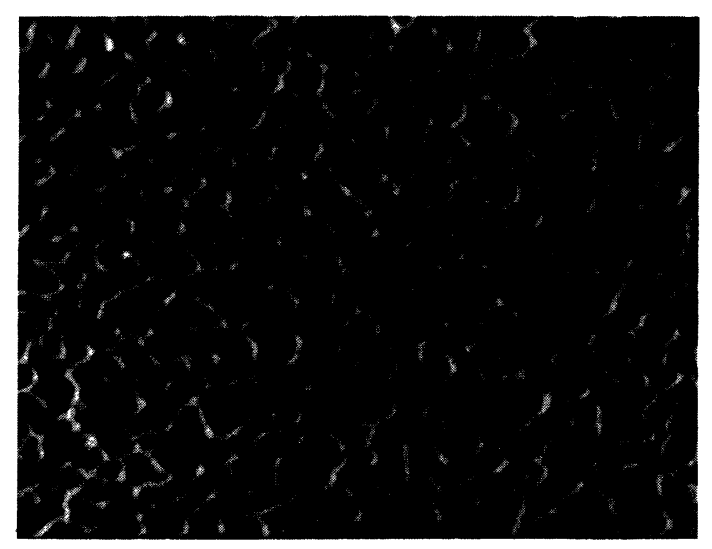

Figure 2c

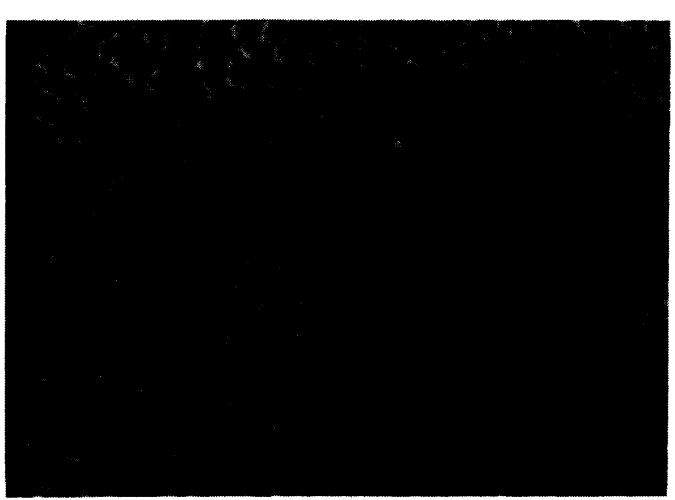

FIGURE 2a'

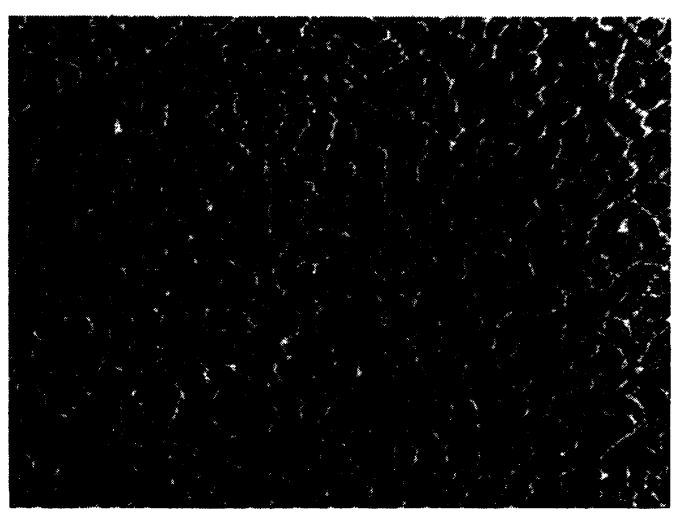

FIGURE $2 b^{\prime}$

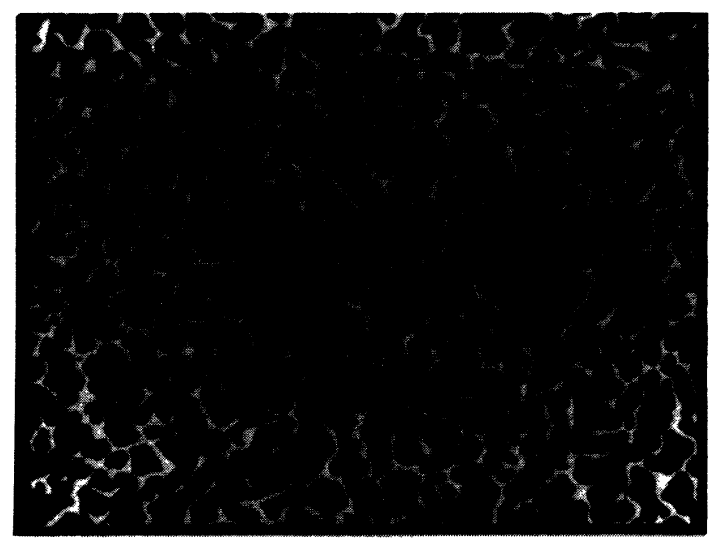

FIGURE 2 $c^{\prime}$

Fig. 2. - Image of a thin granular film (Au). a : image a = original image. $\mathbf{a}^{\prime}:$ image $\mathrm{a}^{\prime}=$ image a, after homogenization and filtering treatments. $b:$ image $b=$ image $a^{\prime}$ after contrast enhancement treatment (Gordon's method). $b^{\prime}:$ image $b^{\prime}=$ image $b$ after thresholding. $c$ : image $c=$ image $a^{\prime}$ after contrast enhancement treatment (modified Gordon's method). $c^{\prime}$ : image $c^{\prime}=$ image $c$ after thresholding. 
details the modifications which we propose to make it suitable to all images. The author defines neighbourhoods by :

$((1,1),(3,3))$ : one pixel surrounded by its eight nearest neighbours; or more generally : $((m, m)$, $(3 m, 3 m)$ ), where $m$ is an odd integer.

If we consider the case $((1,1),(3,3))$, we have the representation :

$$
\begin{array}{lll}
y_{1} & y_{2} & y_{3} \\
y_{4} & y & y_{5} \\
y_{6} & y_{7} & y_{8}
\end{array} .
$$

The local contrast is defined as :

$$
C=\frac{y-\bar{y}}{y+\bar{y}}
$$

with

$$
\bar{y}=\left(y_{1}+y_{2}+y_{3}+y_{4}+y_{5}+y_{6}+y_{7}+y_{8}\right) / 8 \text {. }
$$

We then distinguish two cases :

$$
\begin{aligned}
& y \geqslant \bar{y} \quad \text { so } y=\bar{y} \cdot \frac{1+C}{1-C} \\
& y<\bar{y} \text { and } y=\bar{y} \cdot \frac{1-C}{1+C} .
\end{aligned}
$$

We notice that $C$ is a real satisfying

$$
0 \leqslant C \leqslant 1 .
$$

We can enhance the contrast $C$ by using any function $F$ satisfying :

$$
x \in[0,1]: F(x)[0,1] \text { and } F(x) \geqslant x:
$$

The simplest function is the square root function. So we transform $C$ into $C^{\prime}=\sqrt{C}$.

Therefore $y$ will be transformed into :

$$
y^{\prime}=\bar{y} \frac{1+C^{\prime}}{1-C^{\prime}} \quad \text { if } \quad y \geqslant \bar{y}
$$

or

$$
y^{\prime}=\bar{y} \frac{1-C^{\prime}}{1+C^{\prime}} \text { if } y<\bar{y}
$$

We applied this procedure to all our images and concluded that the result is very sensitive to noise in the case of small neighbourhood $((1,1),(3,3))$ and that the method does not work for others neighbourhoods (see Fig. 2).

Therefore we reconsidered the Gordon algorithm and tried to adapt it to noisy images by taking into account visual perception criteria [4]. It is well known that all perception mechanisms are more sensitive to contours and to the mean gray level associated to sufficiently large areas. Thus we modied the Gordon treatment by applying contour detection operators to windows of adequate size. We subdivise the picture into blocks of windows (same size). The size of these windows must be adequate, like in the correction for illumination inhomogeneity. In each window $W_{i}$, we compute the edge value $\Delta_{j}$, using the Laplacian or the Sobel operator, which are less sensitive to noise [18]. $\Delta_{j}$ is associated to the current pixel of gray level $y_{j}$. Then we select only the pixels having high edge values for computing the main gray edge level (i.e. the main gray level of points lying on the cluster frontiers). For instance with the Laplacian operator, $\Delta_{j}$ is defined by $\Delta_{j}=\left|y_{j}-\bar{y}\right|$, where $y_{j}$ is the gray level of the pixel $x_{k l}\left(y_{j}=f\left(x_{k l}\right)\right)$ and $\bar{y}$ the mean gray level of the eight nearest neighbour pixels of $x_{k l}$.

Therefore :

$$
\bar{e}_{i}=\sum_{x_{k l} \in W_{i}^{*}} e_{j} / \operatorname{dim}\left(W_{i}^{*}\right)
$$

is the mean edge gray level.

$W_{i}^{*}:$ the set of pixels having their high edge value in $W_{i}$.

$x_{k l}$ is a pixel in $W_{i}^{*} W_{i}$, with gray level $y_{j}$.

$e_{j}$ : the edge gray level associated to the pixel $x_{k l}$.

The contrast is then defined as :

$$
C_{i}\left(y_{j}\right)=\left|\frac{y_{j}-\bar{e}_{i}}{y_{j}+\bar{e}_{i}}\right|
$$

and we transform $y_{j}$ into $y_{j}^{\prime}$ like in the Gordon approach. As shown in figure 3 , this method reveals the presence of two classes.

We prefer, to improve, with this method, the intrinsic information of the image rather than that of its histogram, as it is done by some authors [21-23].

4.3 Thresholding methods. - We present now three specific thresholding methods. The first two use the histogram information : the Velasco method [17] is an iterative method; the Otsu method is based on discriminant analysis [28]. With these two methods we obtained the same threshold for all our images. Thus we only describe the Velasco method. The most efficient method is however the third one, due to Dunn et al. [7]. It does not use the gray level histogram but a local analysis.

4.3.1 Description of the Velasco method (isodata clustering algorithm). - This iterative method has been proposed by Ridler and Calvard [26], for the first time, for object-background discrimination. The Velasco method is a generalization of this method to more than two classes. It is useful for data compression without significant distortion of the image as in [27].

The expression of the algorithm in the case of two classes is :

1st : determine the smallest interval containing all non zero histogram values : $[L O, U P]$.

2nd: select an initial threshold $T^{0}=L O+1$.

3rd : set $i=1$ and select some initial values for the mean values $\mu_{1}^{i}$ and $\mu_{2}^{i}$

$$
\left(L O \leqslant \mu_{1}^{i}<\mu_{2}^{i} \leqslant U P\right)
$$



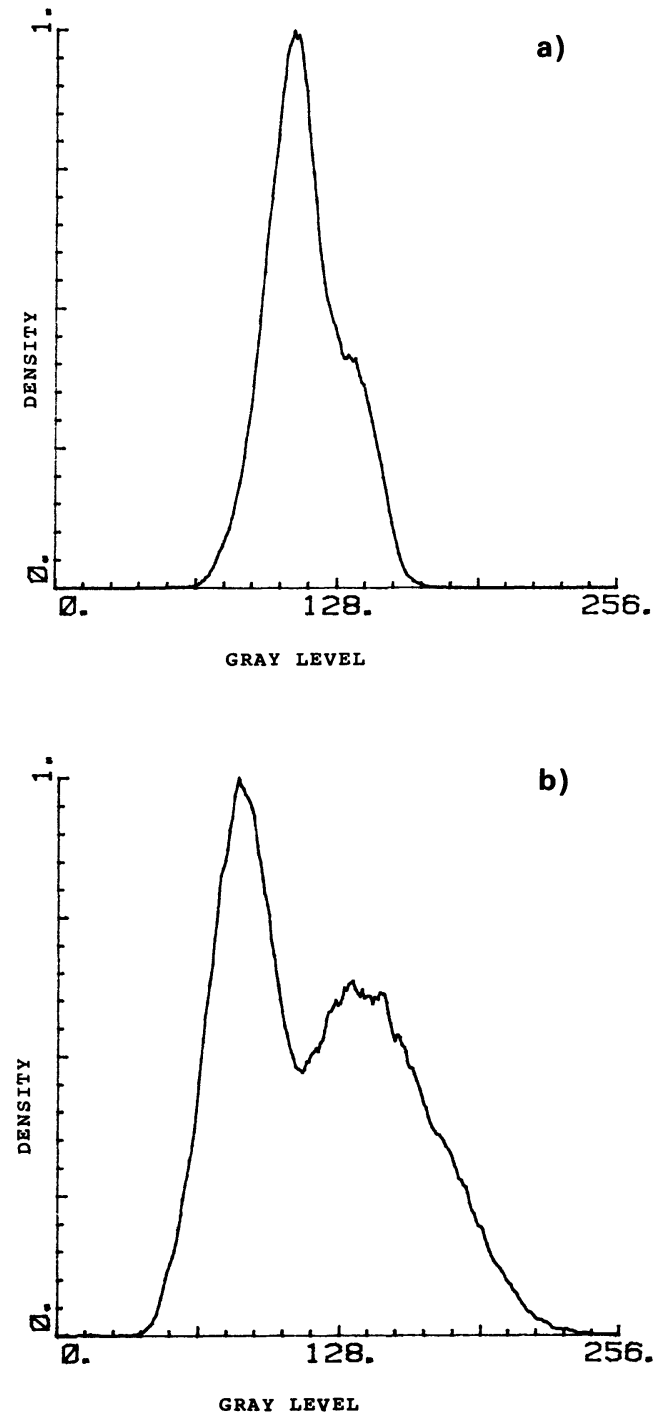

Fig. 3. - Gray level histograms. a : gray level histogram of the original image (cf. Fig. 2a'). b : gray level histogram of the image after contrast enhancement (cf. Fig. 2b).

4th : loop 4 calculate threshold $T^{i}=\frac{\mu_{1}^{i}+\mu_{2}^{i}}{2}$ and assign all gray levels in the interval $I_{1}=\left[L O, T^{i}\right]$ to class $I$ and in the interval $I_{2}=\left[T^{i}+1, U P\right]$ to class II.

5th : recompute the mean values :

$$
\mu_{k}^{i}=\left\{\sum_{j \in J I_{k}^{i}} j . h(j)\right\} /\left\{\sum_{j \in J_{I_{k}^{i}}^{i}} h(j)\right\}
$$

$k=1$ and 2 respectively.

$h$ is the histogram gray level.

6th : if any mean value has changed, go to the 4th loop; otherwise, stop.

Since we have always $L O \leqslant \mu_{l}^{k} \leqslant T^{k}<\mu_{2}^{k} \leqslant U P$, and if $T^{i} \leqslant T^{i+1}$, then $T^{i+1} \leqslant T^{i+2}$, the sequence $T^{0}, T^{1}, \ldots$ of thresholds forms either a non-decreasing or a non-increasing sequence. This is bounded and, therefore $k$ exists such that $T^{k}=T^{k+1}$. If this happens, then $T^{k}=T^{k+j}$ for all $j$ and the threshold is found.

4.3.2 Algorithm of Dunn et al. (Also called the uniform error threshold (UET). - In this method, one selects the threshold equalizing the probability of misclassification in an image containing two classes $A_{1}$ and $\mathrm{A}_{2}$. Let $p_{1}(x)$, and $p_{2}(x)$ be the probability distribution of gray levels in the class $A_{1}$ and the class $A_{2}$ respectively.

Let $\alpha$ be the fraction of $A_{1}$; thus the area of $A_{2}$ is $1-\alpha$.

Let $t$ be a candidate threshold. Pixels with gray level smaller than or equal to $t$ are classified in $A_{1}$ (black class), and pixels with gray level greater than $t$ are classified in $\mathrm{A}_{2}$ (white class). The probability that a point of $A_{1}$ has been misclassified in $A_{2}$ is given by :

$$
\int_{t}^{\infty} p_{1}(x) \mathrm{d} x=1-P_{1}(t)
$$

and the probability of misclassifying a $A_{2}$ point in $A_{1}$ is given by :

$$
P_{2}(t)=\int_{-\infty}^{t} p_{2}(x) \mathrm{d} x
$$

the minimum error thresholding consists in minimizing the total misclassification error :

$$
\alpha . P_{2}(t)+(1-\alpha) \cdot\left[1-P_{1}(t)\right] .
$$

It can be achieved if $P_{1}$ and $P_{2}$ are known [16]. We have shown before how difficult and ambiguous is the fitting of $P_{1}$ and $P_{2}$ by normal distributions.

Dunn follows a more original way : instead of fitting the histogram by two normal distributions $\boldsymbol{P}_{1}$ and $P_{2}$, he examines $2 \times 2$ neighbourhoods. Let us fix a threshold, say $t$.

Let $\alpha_{1}(t)$ denote the fraction of pixels in $A_{1}$ that are " white », i.e. gray levels greater than $t$. Similarly define $\alpha_{2}(t)$ as the fraction of pixels in class $A_{2}$ that are " white " (i.e. : the fraction of misclassified pixels in $A_{1}$ is $\alpha_{1}(t)$ and the fraction of misclassified pixels in $A_{2}$ is $\left.1-\alpha_{2}(t)\right)$. Thus the uniform error threshold is the gray level $t$ such that :

$$
\alpha_{1}(t)=1-\alpha_{2}(t)
$$

i.e. the probabilities of misclassification for $A_{1}$ and $A_{2}$ are equal.

Let us define the three probabilities $a, b$ and $c$ as :

$a=\operatorname{Prob}\{$ pixel has gray level $>t:$ "white» $\}$

$b=\operatorname{Prob}\{2$ adjacent pixels are " white " $\}$

$c=\operatorname{Prob}\{4$ neighbouring pixels are « white $»\}$

so

$$
\left\{\begin{array}{l}
a=\alpha \cdot \alpha_{1}+(1-\alpha) \cdot \alpha_{2} \\
b=\alpha \cdot \alpha_{1}^{2}+(1-\alpha) \cdot \alpha_{2}^{2} \\
c=\alpha \cdot \alpha_{1}^{4}+(1-\alpha) \cdot \alpha_{2}^{4}
\end{array}\right.
$$


the following equation has then to be solved : $\alpha_{1}=1-\alpha_{2}$ or equivalently $\phi-1=0$, where $\phi=$ $\alpha_{1}+\alpha_{2}$. One can express $\phi$ in terms of $a, b$ and $c$ :

$$
\phi^{2}=\frac{b^{2}-c}{a^{2}-c} .
$$

Once the $a, b$ and $c$ probabilities have been estimated from the examination of $2 \times 2$ neighbourhoods in the image (for a given value of $t$ ), (6) can be solved. Now the uniform error threshold is the gray level $t$ verifying :

$$
\phi=1 \text {. }
$$

Practically, $t$ is choosen such that $|\phi-1|<\varepsilon$ for some suitably small error $\varepsilon$. This $t$ value is the threshold gray level (UET), and the binarization can be performed with this value.

If one wants to go further and solve for the minimum error threshold without any assumptions on the distribution, one has to use the previous development. Recall that the minimum error threshold is the gray level $t$ such that :

$$
\alpha . \alpha_{1}+(1-\alpha) .\left(1-\alpha_{2}\right)
$$

is minimized (i.e. the error is weighted by the areas of $A_{1}$ and $A_{2}$ ). One uses equations (5) to solve for $\alpha_{1}$ and then $\alpha_{2}=\phi-\alpha_{1}$. To solve for $\alpha$, recall that $a=\alpha \alpha_{1}+(1-\alpha) \alpha_{2}$ or equivalently,

$$
\alpha=\frac{a-\alpha_{2}}{\alpha_{1}-\alpha_{2}} .
$$

Equation (8) can be used to estimate the error in the classification. Then the research of the gray level minimizing (8) can start.

This procedure is more efficient than the conventional thresholding algorithms (which are based on the histogram). It is based on a local analysis of the image independent of the distribution. So it is not sensitive to the histogram profile.

\section{Verification of the validity criteria.}

The last step of this image treatment method concerns its validity. Has the information contained in the initial micrograph been altered or not ? Two physical criteria can answer this question.

First, one can compare the values of the metal volume fraction deduced from the image treatment and determined directly by Rutherford backscattering or microprobe analysis. The accuracy of this criterion is limited by dimensionality considerations. The image processing gives in fact a surface fraction (2D) while the physical methods concern the volume fraction (3D). In some samples, the two fractions (2D and 3D) can however be very close, when the particles form a monolayer of flat clusters. This is the case of the $\mathrm{Pt}-\mathrm{Al}_{2} \mathrm{O}_{3}$ film presented in figure 1 . The microprobe analysis gives a Pt volume fraction $q=0.43 \pm$ 0.03 , while a surface algorithm applied to the image treated by the three different methods presented above in 4.3 gives $q=0.442$. The Au granular film (Fig. 2) can also fulfil the dimensionality requirements, but since there is only one element $(\mathrm{Au})$, the matrix being air, the physical methods can not work.

A second criterion can be applied to these Au films. It consists in verifying, after image treatment, the connectivity of an infinite cluster throughout the image for samples at the percolation threshold. This test is very sensitive, since the threshold observed on the electrical conductivity is very steep. In the case of $\mathrm{Au}$ films near the electrical percolation, the three thresholding methods indeed show as expected the occurrence of the infinite cluster.

In conclusion, it is not surprising that Velasco, Otu and Dunn methods similarly verify our criteria, since they define the same grey level threshold ( \pm 1 level). But by comparing their results to other data on the same films, we confirm that this thresholding as well as the previous treatment do not affect the information contained in the micrograph.

\section{Conclusion.}

We have shown that it is possible to select an optimum method for a computer study of the TEM micrographs of thin granular films. The image treatment organi-

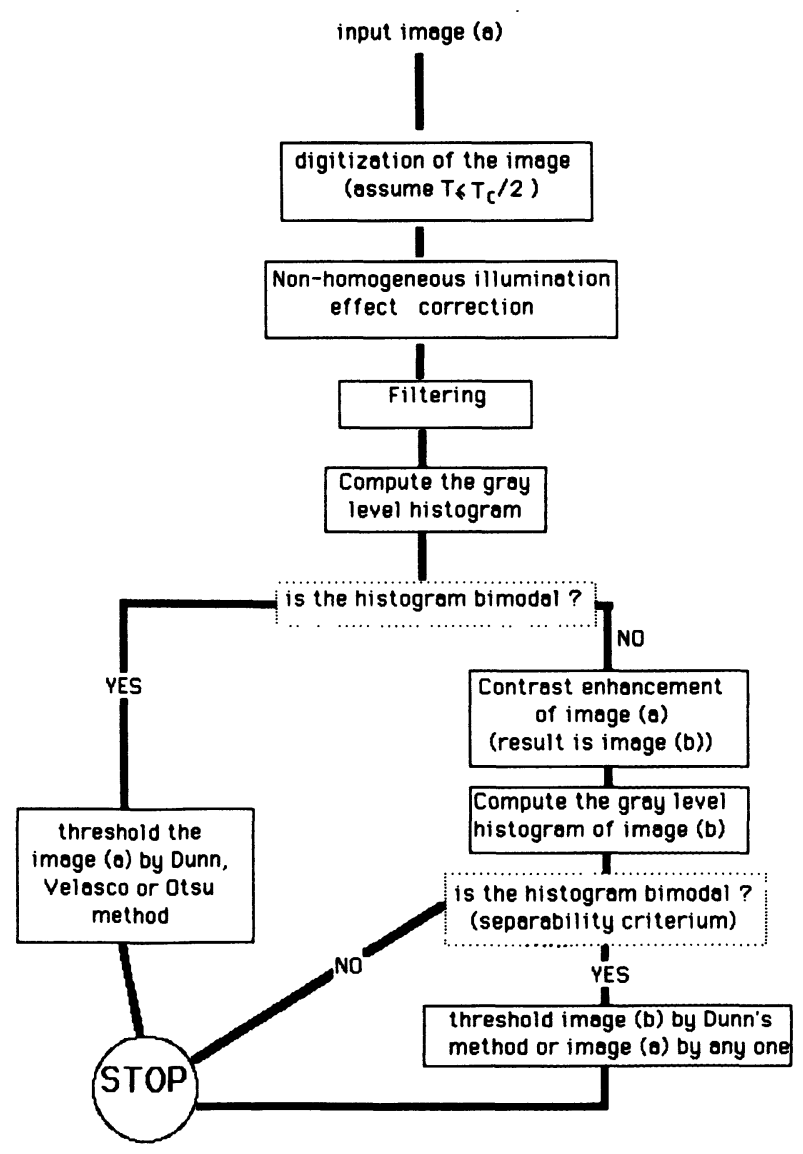

Fig. 4. - Image processing routines. 
gram is schematized in figure 4. It is well known that the choice of the threshold is decisive for any physical interpretation, especially at the metal-insulator transition in granular metallic films, when the infinite cluster [8] needs to be isolated for a statistical study of percolation in the framework of scaling laws. We have developed an efficient image-processing treatment leading to a single unambiguous optimum threshold avoiding the dubiousness encountered by many authors (for instance see Ref. [8]).

\section{Acknowledgments.}

We wish to thank Dr. A. Rosenfeld and Dr. S. Dunn for helpfull epistolary exchanges, O. Bouet and A. Le Negrate for computer and H. Dupoisot for fruitful discussions.

\section{References}

[1] Sella, C., Lafait, J., Berthier, S., Vien, T. K., Thin Solid Films 90 (1982) 425.

[2] Mandelbrot, B. B., The fractal geometry of nature (W. H. Feeman and Co. San Francisco) 1982.

[3] Bittar, A., Berthier, S., LAfait, J., J. Physique 45 (1984) 623.

[4] Rosenfeld, A. and KAK, A., Digital image processing, vol. 1, 2nd edition (Academic Press) 1982.

[5] Gordon, R. and Rangayan, R. M., Appl. Optics 23 (1984) 560.

[6] Pratt, W. K., Digital image processing (Wiley Interscience) 1978.

[7] DunN, S., Private communication.

[8] Kapitulnik, A. and Deutscher, G., Thin Solid Films 113 (1984) 79.

[9] Lowitz, G. E., Pattern Recognition 10 (1978) 351.

[10] Rosenfeld, A., Private communication.

[11] Weszka, J. S. and Rosenfeld, A., IEEE. Trans. Syst. Man. Cybern. 9 (1979) 38.

[12] Voss, R. F., Phys. Rev. Lett. 49 (1982) 1441.

[13] Kapitulnik, A. and Deutscher, G., Phys. Rev. Lett. 49 (1982) 1444.

[14] Kirby, R. L. and Rosenfeld, A., IEEE. Trans. Syst. Man. Cybern. 9 (1979) 860.

[15] Watanabe, S. and the cybest group. Comput. Graphics Image Processing 3 (1974) 350.
[16] OTSU, N., IEEE. Trans. Syst. Man. Cybern. 9 (1979) 62.

[17] Flavio, R. D., Velasco, IEEE. Trans. Syst. Man. Cybern. 10 (1980) 771.

[18] Rosenfeld, A., volume 2 of reference [4].

[19] Granlung, IEEE Trans. Biom. Eng. 10 (1980) 771.

[20] PolaK, E., Computational methods in Optimization (Academic Press) 1971.

[21] Weszka, J. S. and Rosenfeld, A., IEEE. Trans. Syst. Man. Cybern. 8 (1978) 622.

[22] Durga, P. P. and Rosenfeld, A., IEEE. Trans. Comput. 27 (1978) 875.

[23] Ahuja, N. and Rosenfeld, A., IEEE. Trans. Syst. Man. Cybern. 8 (1978) 895.

[24] FreI, W., Comput. Graphics Image Processing 6 (1977) 286.

[25] Humel, R., Comput. Graphics Image Processing 6 (1977) 184.

[26] Ridler, T. W. and Calvard, S., IEEE. Trans. Syst. Man. Cybern. 8 (1978) 630.

[27] Peleg, S., IEEE. Trans. Syst. Man. Cybern. 8 (1978) 555.

[28] Fukunaga, K., Introduction to statistical pattern recognition (New-York, Academic Press) 1972, p. 260-267. 